\title{
Calidad de Andosols en sistemas forestal, agroforestal y agrícola con diferentes manejos en Zacatlán, Puebla
}

\author{
The quality of Andosols under forestry, agroforestry and agricultural management in Zacatlán, Puebla
}

\author{
Jonathan Omar Hernández Ordoñez ${ }^{1}$, Ma. del Carmen Gutiérrez Castorena ${ }^{1:}$ \\ Carlos Alberto Ortiz Solorio', Patricio Sánchez Guzmán ${ }^{1}$ y Efraín Ángeles Cervantes²
}

\footnotetext{
${ }^{1}$ Área de Génesis, Morfología y Clasificación de Suelos, Edafología. Colegio de Postgraduados, Campus Montecillo. Km 36.5 Carretera México-Texcoco. 56230 Texcoco, Estado de México, México.

*Autora responsable (castor@colpos.mx)

${ }^{2}$ Laboratorio de Eco-hidrología, UMIEZ, FES Zaragoza, UNAM. Batalla 5 de Mayo SN, Iztapalapa, Ejercito Oriente. 09230 Ciudad de México, CDMX.
}

\section{RESUMEN}

Los estudios de suelos en sistemas agroforestales relacionados con bosques mesófilos de montaña han sido poco abordados en nuestro país, especialmente los desarrollados en Andosols, los cuales son susceptibles a los procesos de degradación por el cambio de uso del suelo. El presente estudio se realizó en el municipio de Zacatlán, Puebla con el objetivo de caracterizar la calidad de los Andosols bajo diferentes sistemas de manejo. Los métodos que se emplearon fueron: entrevistas a productores para identificar los sistemas, descripción de perfiles de suelos, análisis de laboratorio y análisis de similaridad de los diferentes manejos. Los resultados indicaron que la zona de estudio está integrada por tres sistemas: bosque mesófilo de Montaña (BMM), agroforestal (asociaciones manzanamaíz-calabaza-arándanos) y agrícola, con adición de abonos orgánicos (FO) o fertilización inorgánica (FI). En los Andosols con BMM, la estructura biológica domina (agregados migajosos y granulares hasta los $55 \mathrm{~cm}$ de profundidad), presentan alto contenido de materia orgánica del suelo (MOS) (31\%), alta retención de humedad (194.5\%) y baja densidad aparente (Da) $\left(0.49 \mathrm{~g} \mathrm{~cm}^{-3}\right)$. Con el cambio de uso de suelo (de bosque a agroforestal o agrícola), la estructura biológica se reduce en espesor o desaparece y dominan los bloques sub-angulares, debido a la disminución drástica del contenido de MOS (a 16.3 o a $6.3 \%$ ), y retención de humedad (de 97 o a $47 \%$ ). Además, la Da aumentó hasta $1 \mathrm{~g} \mathrm{~cm}^{-3}$ en el sistema agrícola. Por el contrario, en tierras degradadas, el sistema agroforestal con manejo orgánico ha mejorado la calidad del horizonte superficial en menos de seis

Cita recomendada:

Hernández Ordoñez, L. O., M. del C. Gutiérrez Castorena, C. A. Ortiz Solorio, P. Sánchez Guzmán y E. Ángeles Cervantes. 2017. Calidad de Andosols en sistemas forestal, agroforestal y agrícola con diferentes manejos en Zacatlán, Puebla. Terra Latinoamericana 35: 179-189. años y con la FO o FI algunos sistemas agroforestales aumentaron los contenidos de $\mathrm{N}_{\text {tot }}$ y P. Los índices que mejor caracterizaron la calidad de los Andosols bajo diferentes sistemas fueron: estructura del suelo, MOS, $\mathrm{N}_{\text {tot }}$, P y retención de humedad.

Palabras clave: bosque mesófilo, manejo orgánico e inorgánico, estructura del suelo.

\section{SUMMARY}

Soil studies on agroforestry systems related to cloud forests have been poorly studied in our country, especially those systems that develop in Andosols, which are very susceptible to land degradation processes with changes in land use. The present study was carried out in the municipality of Zacatlán, Puebla, to characterize the quality of Andosols under different management systems. The methods used were interviews with producers to identify the systems, description of soil profiles, laboratory analysis and similarity analysis of different management systems. In addition, with edaphic information, quality indices of the different management systems were established. The results indicate that the study area is composed of three systems: cloud forest, agroforestry (apple-blueberry-pumpkin-maize-associations) and agricultural management, with addition of organic and inorganic fertilization (OF and IF, respectively). In Andosols under cloud forests, the biological structure is dominant (crumb texture and granular aggregates, up to $55 \mathrm{~cm}$ deep). Furthermore, they have high contents of soil organic matter (31\%), high moisture retention (194.5\%), and low bulk density $0.49 \mathrm{~g} \mathrm{~cm}^{-3}$. 
With land use change (to agroforestry or agricultural), the biological structure is reduced in thickness or even disappears from the topsoil, and sub-angular blocks dominate, due to the drastic decrease in organic matter content (16.3 to 6.3\%), and water retention (97 to $47 \%$ ). In addition, bulk density increases from 0.49 to $1 \mathrm{~g} \mathrm{~cm}^{-3}$ in the agricultural system. Conversely, in degraded lands, agroforestry systems with organic fertilization has improved the quality of the surface horizon in less than six years, and with organic or inorganic fertilization some agroforestry systems increase the contents of total $\mathrm{N}$ and $\mathrm{P}$. The indexes that best characterize the quality of the Andosols under different systems are soil structure, soil organic matter, $\mathrm{N}_{\text {tot }}$, and moisture retention.

Index words: cloud forest, organic and inorganic fertilization, soil structure.

\section{INTRODUCCIÓN}

En México, el manejo agroforestal se ha centrado principalmente en zonas tropicales y templadas, (Moreno-Calles et al., 2013), en donde se presentan los bosques mesófilos de montaña (BMM). Estos ecosistemas cubren una superficie entre $0.4-1 \%$ del territorio nacional, presentan alta biodiversidad (Villaseñor, 2010; Fa y Morales, 1993) y gran variedad de servicios ecosistémicos (Martínez et al., 2009). Sin embargo, el avance de la frontera agrícola hacia estas superficies forestales ha traído como consecuencia la aparición de una superficie de labor frágil, con alta susceptibilidad a los procesos erosivos y, por ende, una baja productividad (Orozco et al., 2004).

Los sistemas agroforestales pueden ser una alternativa para la agricultura en los bosques mesófilos pues ofrecen beneficios económicos, sociales y ambientales (Young, 1989 y 1997; CATIE, 2001; USDA, 2014; Wilson y Lovell, 2016), así como la reducción del uso de insumos (Strong y Jacobson, 2006). No obstante, han sido cuestionados por su baja productividad económica a corto plazo, se aplican sólo en áreas pequeñas y los resultados no son fácilmente reproducibles por las condiciones ambientales específicas en donde se han realizado (CATIE, 2001; Moreno-Calles et al., 2013). Además, estos estudios se basan en la evaluación de la producción de biomasa y alimentos, servicios ecosistémicos, calidad del aire y del agua (Casanova-Lugo et al., 2016; Torralba et al.,
2016), pero poco se analiza su manejo de manera integral, donde se consideren factores clave como la calidad del suelo e indicadores como la agregación, importante en la estabilización del carbono.

En la región de Zacatlán, Puebla, los BMM forman parte de la Región Terrestre Prioritaria de Conservación RTP 102, y presentan dominantemente Andosols (Krasilnikov et al., 2013). Esta región muestra fragmentación del hábitat en $60 \%$ e intensa degradación de suelos (SEMARNAP-CP, 2002). Un diagnóstico de los sistemas (o del impacto del cambio de uso de suelo) son importantes para proponer estrategias que mitiguen el impacto de las prácticas agrícolas y ganaderas, y con ello, lograr la conservación de los BMM y sus servicios ecosistémicos (Arriaga et al., 2000).

Los Andosols constituyen uno de los grupos de suelos de referencia para la producción agrícola en el mundo por su elevada fertilidad potencial (IUSS Working Group WRB, 2015). La relación que tienen con zonas volcánicas permiten actividades forestales, agrícolas y frutícolas bajo diferentes manejos tanto orgánicos como inorgánicos, y con diferentes sistemas de labranza: convencional, tradicional, mínima o cero (Tapia-Vargas et al., 2001; Velásquez-Valle et al., 2006, 2007; Muñoz-Villalobos et al., 2011). El problema de estos suelos es que son altamente susceptibles a la erosión hídrica y eólica (Krasilnikov et al., 2013), lo que incrementa aún más la fragilidad de los ecosistemas de los BMM. La hipótesis que se plantea en la presente investigación es que los sistemas agroforestales en BMM son adecuados para la conservación o mejoramiento de la calidad de los Andosols después del cambio de uso de suelo; no obstante, puede haber diferencias entre los manejos orgánicos e inorgánicos en sus propiedades edáficas.

El objetivo principal del estudio fue evaluar la calidad de los Andosols bajo diferentes sistemas: forestal, agroforestal y agrícola con diferentes manejos, en Zacatlán, Puebla.

\section{MATERIALES Y MÉTODOS}

El Municipio de Zacatlán se localiza dentro de la región de la Sierra Norte, del estado de Puebla, entre los paralelos $19^{\circ} 50^{\prime} 06^{\prime \prime}$ y $20^{\circ} 08^{\prime} 12^{\prime \prime}$ de N y los meridianos $97^{\circ} 51^{\prime}$ '06" y $98^{\circ} 12^{\prime} 36^{\prime \prime} \mathrm{O}$; presenta una elevación que varía de 1120 a $2860 \mathrm{~m}$. El clima es templado húmedo con abundantes lluvias en verano (temperatura media anual de $14.5^{\circ} \mathrm{C}$ y con 
precipitaciones medias anuales de $1073 \mathrm{~mm}$ ); la vegetación está dominada principalmente por BMM y bosque de pino-encino que pertenecen a la Región Terrestre Prioritaria de Conservación RTP 102 (Arriaga et al., 2000). Los suelos predominantes son Andosols, aunque también pueden ocurrir Luvisols y Cambisols de acuerdo con la información del Plan de Desarrollo Municipal (H. Ayuntamiento de Zacatlán, 2011).

Los sitios de muestreo se ubicaron en dos comunidades de Zacatlán: Atzingo y Poxcuatzingo, los cuales se localizan al norte de la cabecera municipal. A través de recorridos de campo se identificaron sitios en diferentes sistemas: forestal (Testigo), agroforestal y agrícola. Posteriormente, se entrevistaron a comisariados ejidales y productores para conocer el manejo agronómico (adición de abonos orgánicos o aplicación de fertilizantes inorgánicos) y el tipo de labranza (mínima o cero y convencional); además se cuestionó si los suelos habían presentado algún tipo de degradación. En cada sitio se realizaron pozos pedológicos con diferentes profundidades y se describieron de acuerdo con el manual de Cuanalo (1990). Las muestras de suelo fueron colectadas en cada uno de los horizontes; además, se obtuvieron muestras compuestas (tres sub-muestras) de la capa superficial (0 a $20 \mathrm{~cm}$ ) de cada sistema de manejo, para su posterior análisis en el laboratorio.

Las muestras alteradas se secaron a temperatura ambiente y se tamizaron con una malla de $2 \mathrm{~mm}$ de apertura para separar la fracción de tierra fina del suelo. Las determinaciones químicas que se realizaron fueron: reacción del suelo ( $\mathrm{pH}$ relación agua suelo 2:1); Nitrógeno total $\left(\mathrm{N}_{\text {tot }}\right)$ por el método de Kjeldahl, Materia Orgánica del Suelo (MOS) con el método de Walkley y Black; Fósforo soluble en ácido cítrico $\left(\mathrm{P}_{\mathrm{ac} \text { cit }}\right)$; y Capacidad de Intercambio Catiónico (CIC) con el método del acetato de amonio. Las determinaciones físicas fueron: densidad aparente (Da), con el método del terrón parafinado; Textura con el método de la pipeta americana, Retención de Humedad (Membrana y olla de presión) y Color (Carta de color Munsell). Todos los procedimientos fueron extraídos del manual de van Reeuwijk (2003) y fueron realizados con tres repeticiones.

La calidad de suelo de cada sistema analizado se obtuvo a través de índices de acuerdo con Larson y Pierce (1994) y Bautista-Cruz et al. (2004). Con los datos de las propiedades edáficas de cada sistema se realizó un análisis de similaridad utilizando una codificación $0-1$, donde 0 se asigna al valor mínimo y 1 al valor máximo de cada propiedad y se usó la distancia euclidiana.

\section{RESULTADOS Y DISCUSIÓN}

El análisis de similaridad de las propiedades del suelo mostró que en la zona de estudio se presentaron tres grupos: forestal (BMM), agroforestal (con manejo orgánico y labranza mínima) y agrícola (manejo inorgánico y labranza convencional). El grupo o sistema que dominó fue el agroforestal. En la Figura 1 se muestra el paisaje de cada sistema estudiado.

En los sistemas agroforestales destacan las plantaciones de manzana, arándanos, cultivo de maíz; además de asociaciones de maíz-habacalabaza con árboles de manzana. El tiempo durante el cual se ha realizado el manejo de estos suelos es variado, desde 2 hasta 50 años implementando mecanismos de abonamiento similares. En general, la producción de estos sistemas agroforestales se destina para autoconsumo; pocos son los casos en donde la producción y los intereses del propietario son con fines comerciales. El manejo agrícola está reservado principalmente a la producción de maíz para autoconsumo. En el Cuadro 1 se reportan los sistemas estudiados y sus principales características.

\section{Propiedades Edáficas}

La estructura del suelo es una de las propiedades que más se relaciona con otras propiedades físicas y químicas como: color, MOS, porosidad, retención de humedad y densidad aparente (Brady y Weil, 1999), de ahí la importancia de su análisis a nivel de perfil de suelos en los diferentes sistemas estudiados. En la Figura 2 se reportan diferentes grados de descomposición de la materia orgánica (a y b) y la formación de agregados en el horizonte orgánico y superficial (c y d); mientras que en la Figura 3 se ilustran los diferentes tipos de agregados del suelo que se presentaron en los sistemas estudiados.

En el BMM, los agregados migajosos y granulares son abundantes desde la superficie hasta profundidades de 55 y $70 \mathrm{~cm}$, respectivamente (Sitio 1). Este tipo de agregados son comunes en los horizontes orgánicos y superficiales como resultado de la actividad biológica 


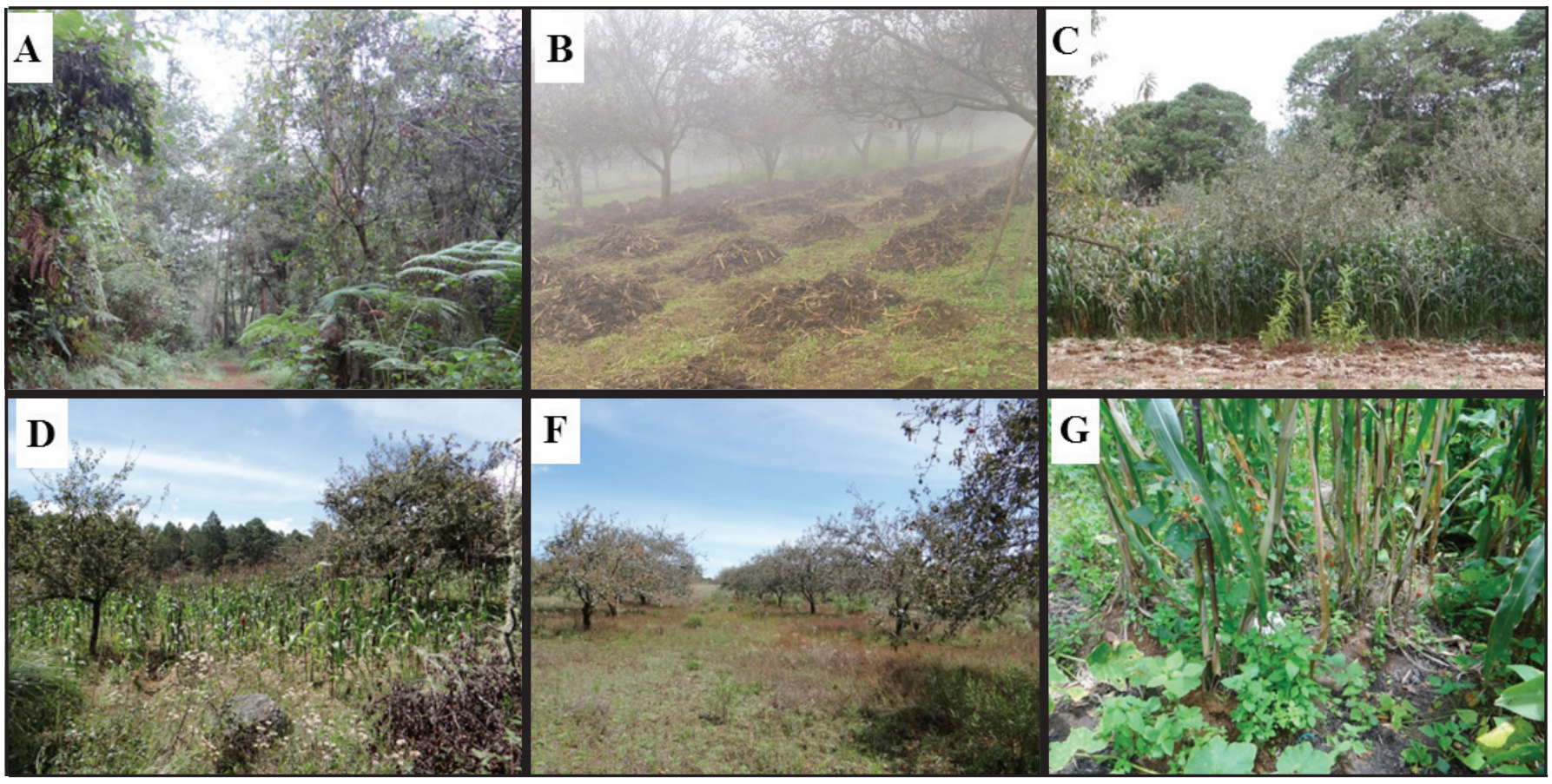

Figura 1. (A) Bosque mesófilo de montaña (BMM); (B) Sistema agroforestal: con enmiendas orgánicas; (C) Agrícola con cero labranza; (D) Agrícola con labranza mínima; (F) Huerto de manzana abandonado; (G) Asociaciones maíz-frijol- haba-calabaza-árboles frutales.

Cuadro 1. Manejo de los sistemas presentes en Zacatlán, Puebla.

\begin{tabular}{|c|c|}
\hline Sitio & Manejo \\
\hline & Bosque Mesófilo de Montaña \\
\hline \multirow[t]{2}{*}{1} & $\begin{array}{l}\text { BMM; gran cantidad de herbáceas y espesor de hojarasca de } 12 \mathrm{~cm} \text {; la actividad de la fauna del suelo y mamíferos medianos } \\
\text { es notable. }\end{array}$ \\
\hline & Sistema Agroforestal (Manejo orgánico) \\
\hline 2 & $\begin{array}{l}\text { Cultivo de maíz y árboles de manzana; abonado con estiércol y cáscara de semilla de calabaza; incorporado con arado animal; } \\
30 \text { años; autoconsumo. }\end{array}$ \\
\hline 3 & $\begin{array}{l}\text { Asociación maíz-haba-manzano entre surcos; abonado con composta a base de fruta, estiércol, zacate, etc.; } 2 \mathrm{Mg}^{-1} \text { anuales } \\
\text { de maíz; incorporada con arado somero; } 2 \text { años; autoconsumo. }\end{array}$ \\
\hline 4 & $\begin{array}{l}\text { Plantación de arándanos; abonado con estiércol de borrego y caballo; no se incorpora, sólo es superficial; } 3 \text { kg por mata; } 6 \\
\text { años; comercial. }\end{array}$ \\
\hline 5 & $\begin{array}{l}\text { Asociación maíz-haba-manzano; abonado con estiércol, lombrices, aserrín y cáscara de semilla de calabaza; incorporado con } \\
\text { maquinaria ligera; } 15 \text { años; comercial. }\end{array}$ \\
\hline 6 & Plantación de manzana y fresa o frutilla silvestre; abonado y tiempo desconocido; labranza cero; comercial. \\
\hline \multirow[t]{2}{*}{7} & Asociación maíz-haba-calabaza; abonado de manera irregular. Autoconsumo. \\
\hline & Sistema Agroforestal (Manejo convencional) \\
\hline 8 & Plantaciones de manzano enano; fertilización química; 50 años; ocho años sin uso; comercial. \\
\hline \multirow[t]{2}{*}{9} & Cultivo de maíz entre árboles de manzana; fertilización química; 2 años de manejo; labranza cero; autoconsumo. \\
\hline & Sistema Agrícola (Manejo convencional) \\
\hline 10 & $\begin{array}{l}\text { Cultivo de maíz y bosque; cultivo de maíz; fertilización química ocasional; tiempo desconocido; labranza convencional; } \\
\text { autoconsumo. }\end{array}$ \\
\hline
\end{tabular}



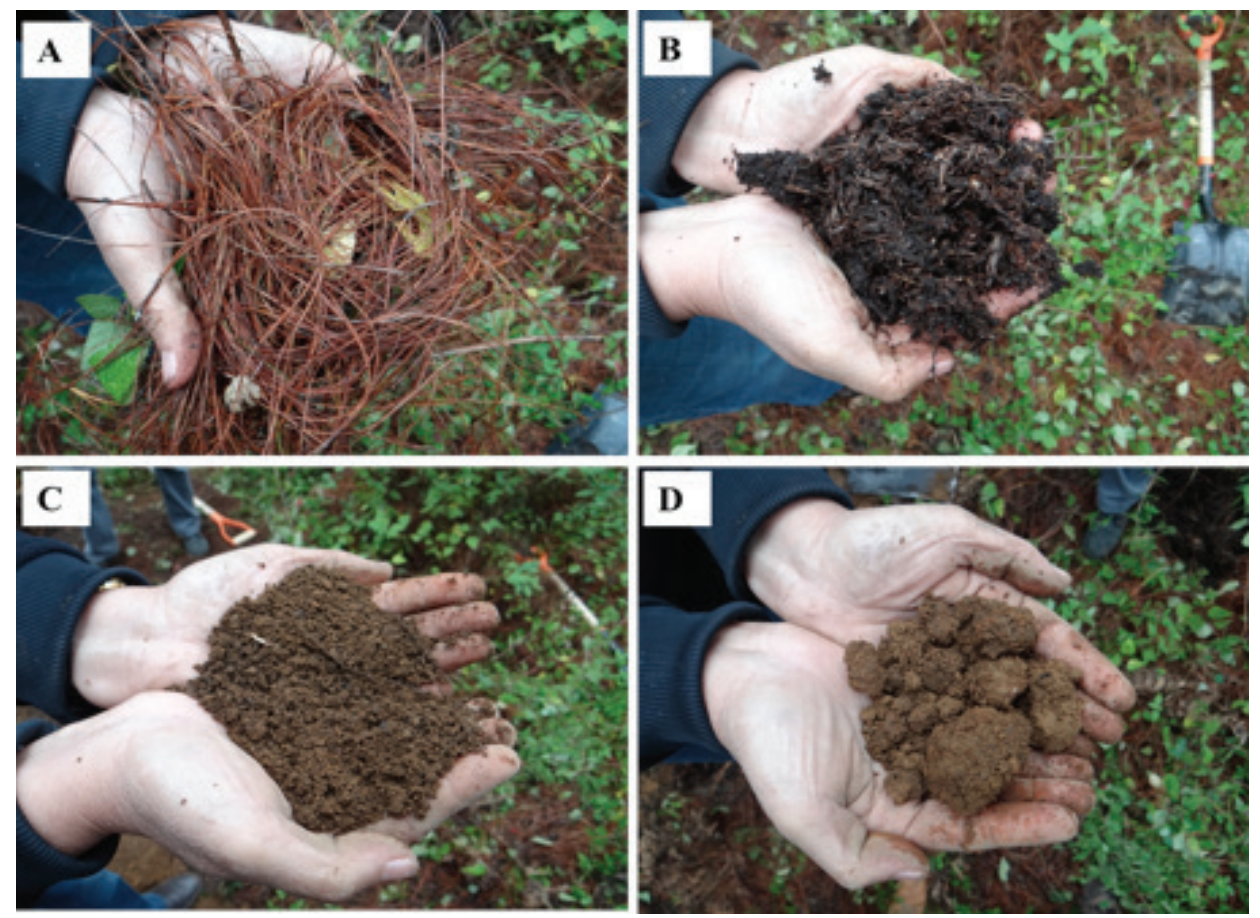

Figura 2. Desarrollo estructural de Andosols bajo BMM. A) y B) Horizonte orgánico con diferente grado de descomposición; C) y D) agregados migajosos y bloque subangulares.

de los hongos que descomponen la materia orgánica, o bien, por excretas de fauna del suelo (Bullock et al., 1985; Bronick y Lal, 2005; Kooistra y Pulleman, 2010), que se acumulan debajo de la hojarasca y superficie del suelo. El gran espesor de los agregados biológicos indica que el BMM no ha sido perturbado.

Con el cambio de uso de suelo (de BMM a manejo orgánico), la frecuencia de los agregados migajosos disminuyó hasta $50 \%$ (Sitios 2 y 3 ) o inclusive no se presentan en suelos con manejo inorgánico (Sitio 9). Sin embargo, los agregados de origen biológico se pueden desarrollar rápidamente en sistemas agroforestales cuyos suelos estuvieron degradados (Sitio 4). Tavares Filho y Tessier (2009) observaron que a una profundidad de 0 a $20 \mathrm{~cm}$, la labranza convencional destruyó a los microagregados, debido a que el manejo del suelo aceleró el proceso de degradación de la MOS (Brady y Weil, 1999).

Los agregados granulares con bloques subangulares son comunes en el sistema agrícola (Sitios 9 y 10), como resultado de la labranza convencional (GutiérrezCastorena et al., 1999), pero también se pueden formar los agregados granulares por la alteración de las cenizas volcánicas de los Andosols (Stoops, 1983; Torn et al., 1997; Stoops et al., 2006). Pagliai et al. (2004) mencionan que la estructura es una de las propiedades del suelo que se modifica rápidamente por el cambio de uso de suelo y por el sistema de labranza.

Otra característica importante que se observó en el sistema agroforestal y agrícola fue la estructura de bloques subangulares cerca de la superficie (manejo agrícola) o entre 15 y $35 \mathrm{~cm}$ de profundidad (Figura 4). Esto implica una degradación de la estructura por disminución en el contenido de MOS (Bronick y Lal, 2005), tanto en labranza convencional como en labranza cero (Cássaro et al., 2011), debido a que esta estructura en Andosols ocurre en los horizontes subsuperficiales (McDaniel et al., 2012). La actividad biológica relacionada con la formación de estos agregados está relacionada sólo con las raíces, las cuales son las responsables del redondeo de los bloques (Brady y Weil, 1999).

El análisis de la estructura cuando ocurre cambio de uso de suelo permite establecer patrones de impacto a nivel de agregación, la cual en el contexto del cambio climático global significa un aspecto importante en el secuestro de carbono, y por ende en la gestión del recurso suelo (Shrestha et al., 2004). No obstante, en estudios de sistemas de agroforestería en nuestro país, este aspecto es generalmente subestimado. 


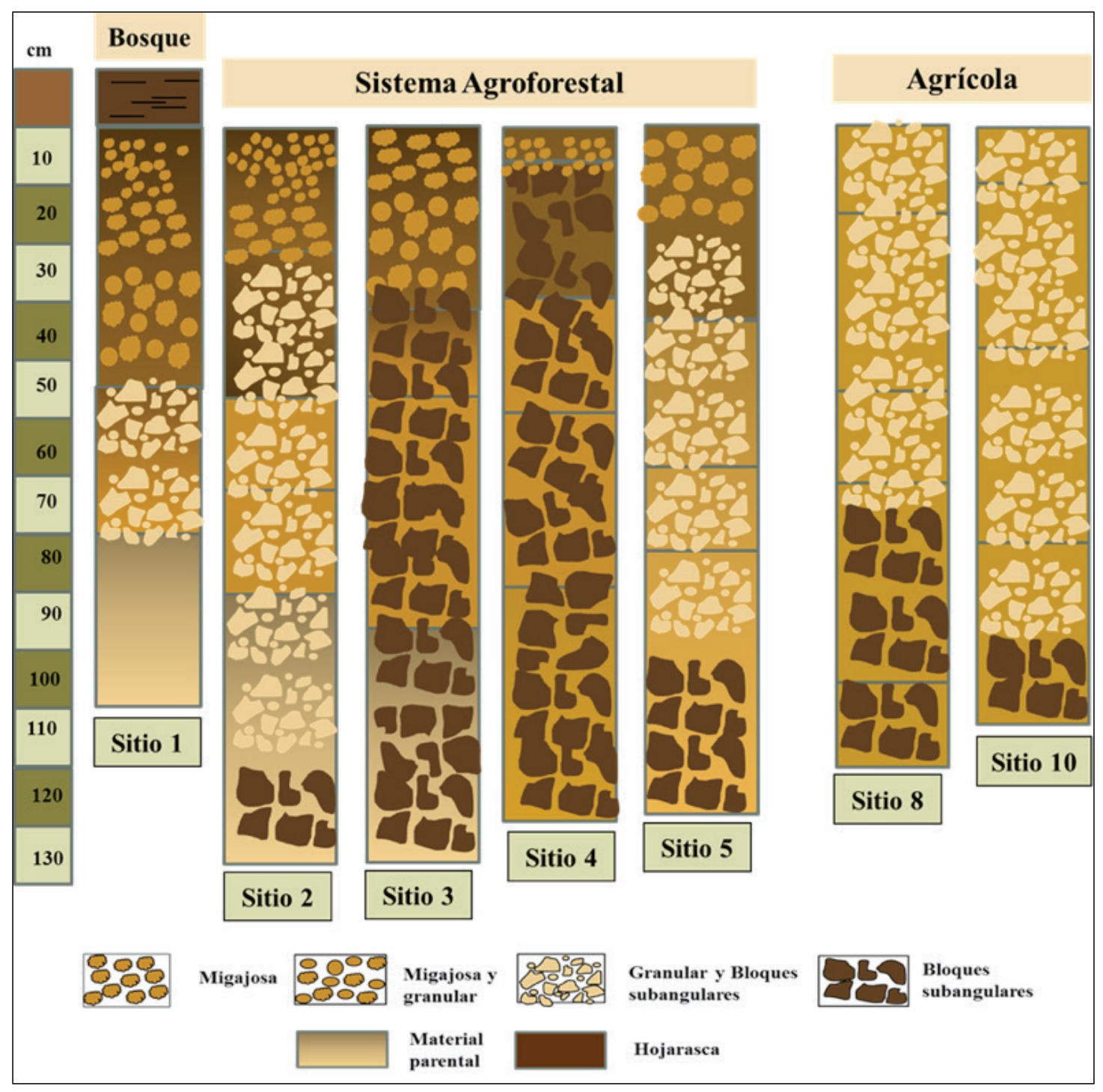

Figura 3. Tipos de agregados presentes en los diferentes sistemas estudiados.

Con la modificación de la estructura, la densidad aparente baja de los Andosols con BMM $\left(0.49 \mathrm{~g} \mathrm{~cm}^{-3}\right)$ comenzó a incrementarse regularmente $\left(0.69 \mathrm{~g} \mathrm{~cm}^{-3}\right)$ (sistema agroforestal con manejo orgánico; Sitio 3), hasta $1 \mathrm{~g} \mathrm{~cm}^{-3}$ (sistema agrícola; Sitio 10). El mismo comportamiento sucedió con la retención de humedad, al disminuir el porcentaje hasta $47 \%$ (Sitio 2) e incluso a 24\% en el manejo convencional (Sitio 8). La densidad aparente baja, alta capacidad de retención de humedad y altos contenidos de amorfos y materia orgánica son comunes en Andosols (IUSS Working Group WRB, 2015); sin embargo, éstas propiedades pueden sufrir cambios irreversibles (McDaniel et al., 2012), por eliminación de la cubierta vegetal o por los diferentes sistemas de labranza. Pires et al. (2017) reportaron que la porosidad total puede disminuir hasta 40\% (0 a $30 \mathrm{~cm}$ de profundidad) de suelos sin labranza a suelos con labranza tradicional.

Finalmente, la textura en la mayoría de los suelos se considera franca limosa y franca; los colores son oscuros en la superficie por el alto contenido de materia orgánica y tienden a ser claros en los horizontes subsuperficiales principalmente en el bosque y en los sistemas agroforestales. En los suelos con manejo agrícola los colores son principalmente claros.

En lo que respecta a las propiedades químicas (Cuadro 2), los cambios más drásticos se presentan en el contenido de MOS, $\mathrm{N}_{\text {tot }}$ y P. El BMM presentó 
Cuadro 2. Propiedades físicas y químicas del horizonte superficial $(0-20 \mathrm{~cm})$ de los sistemas forestales, agrícola y agroforestales de Zacatlán, Puebla.

\begin{tabular}{|c|c|c|c|c|c|c|c|c|c|c|c|}
\hline Manejo/Sitio $^{\dagger}$ & $\mathrm{pH}$ & $\mathrm{C} / \mathrm{N}$ & MOS & $\mathrm{N}_{\text {tot }}$ & $\mathrm{K}+$ & $\mathrm{Ca}^{2+}$ & $\mathrm{Mg}^{2+}$ & $\mathrm{CIC}$ & $\mathrm{P}_{\text {disp }}$ & Humedad & $\mathrm{Da}$ \\
\hline & & \multicolumn{3}{|c|}{$-\ldots-\ldots-\ldots$} & \multicolumn{3}{|c|}{ - - - cmol (+) $\mathrm{kg}^{-1}-$} & - - & $m g \mathrm{~kg}^{-1}$ & $\%$ & $\mathrm{~g} \mathrm{~cm}^{-3}$ \\
\hline \multicolumn{12}{|c|}{ Bosque mesófilo de montaña } \\
\hline 1. Bosque & 4.8 & 100 & 31.1 & 0.18 & 1.14 & 7.87 & 2.97 & 54.4 & 3.1 & 194.1 & 0.49 \\
\hline \multicolumn{12}{|c|}{ Agroforestal (manejo orgánico) } \\
\hline 2. Mz-Mnz-30 & 4.9 & 18.2 & 16.3 & 0.52 & 0.18 & 1.97 & 0.07 & 38.4 & 3.1 & 91.7 & 0.78 \\
\hline 3. Mz-Mnz-Hb-15 & 5.8 & 35.7 & 13.6 & 0.22 & 109.65 & 4.85 & 1.82 & 43.2 & 6.4 & 61.2 & 0.79 \\
\hline 4. B1-3 & 5.2 & 20.9 & 16.9 & 0.47 & 0.3 & 0.43 & 0.05 & 37.8 & 3.8 & 90.8 & 0.7 \\
\hline 5. Mz-Hb-2 & 5.3 & 17.8 & 18.2 & 0.59 & 0.26 & 1.14 & 0.05 & 39.4 & 3.6 & 88.2 & 0.69 \\
\hline 6. Mnz-ND & 6.2 & 20.5 & 13.8 & 0.39 & 1.37 & 2.59 & 1.09 & 35.7 & 3.5 & 58.8 & 0.79 \\
\hline 7. Mz-Hb-Cal-ND & 5.4 & 28.3 & 17.1 & 0.35 & 0.86 & 1.61 & 0.73 & 38.9 & 10.7 & 57.7 & 0.88 \\
\hline \multicolumn{12}{|c|}{ Agroforestal (manejo tradicional abandonado) } \\
\hline 8. Mnz-50 & 6.1 & 20.3 & 11.9 & 0.34 & 1.68 & 4.2 & 0.18 & 31.6 & 3.6 & 54.7 & 0.81 \\
\hline \multicolumn{12}{|c|}{ Agrícola (manejo inorgánico o tradicional) } \\
\hline 9. Mnz-2 & 5.7 & 16.0 & 6.3 & 0.23 & 98.05 & 4.96 & 0.72 & 40.6 & 4.1 & 50.4 & 0.85 \\
\hline 10. Mz-ND & 5.7 & 21.8 & 7.2 & 0.19 & 0.54 & 2.13 & 0.05 & 21.5 & 2.9 & 47.6 & 1.00 \\
\hline
\end{tabular}

Se menciona el: \#sitio-asociación-años de manejo y ND = no determinado; Mz = maíz; Mnz = manzana; Hb = haba; Cal = calabaza; Bl = blue berry.

los valores más altos en MOS (31.1\%) en el horizonte A; mientras que en el sistema agroforestal se redujo hasta $50 \%$ en manejo orgánico (Sitios 2 y 7 ) y hasta $80 \%$ en el sistema agrícola (Sitios 9 y 10). Esos resultados son similares a lo reportado por varios autores cuando hay cambio de uso del suelo (Brady y Weil, 1999; Spaccini et al., 2001; Lal, 2002; Nogueira et al., 2006; Cardoso et al., 2013). Sin embargo, hay que indicar que del bosque nativo a los sistemas agroforestales, el contenido de MOS continua siendo alto (más de 13\%) de acuerdo con la escala de Brady y Weil (1999). Otros autores mencionan que a pesar de que el reemplazo de los bosques por sistemas agroforestales reduce el contenido de MOS, este tipo de sistemas ofrecerá mejores opciones de almacenamiento de carbono que las tierras agrícolas intensamente cultivadas (Nair et al., 2009; Sharma et al., 2016).

La relación $\mathrm{C}: \mathrm{N}$ presente en todos los sitios de estudio fue variable de acuerdo con el bosque y con el tipo de manejo del suelo. Los valores más altos se presentaron en el BMM (C/N de 100), por la presencia de acículas que poseen más lignina, material resistente a la descomposición (Hammel, 1997; Rahman et al., 2013). Con el cambio de uso del suelo e incorporaciones de compostas, las relaciones $\mathrm{C}: \mathrm{N}$ disminuyen entre 16 y 28, comunes en este tipo de materiales orgánicos (Sánchez et al., 2008; Bernal et al., 2009; RomeroFigueroa et al., 2015). En tanto, el Sitio 5 presenta los contenidos de $\mathrm{N}_{\text {tot }}$ y MOS más altos del manejo orgánico ( 0.52 y $18.2 \%$ respectivamente), por el cultivo frecuente de haba, leguminosa que fija nitrógeno (Brady y Weil, 1999); no obstante, también se encontró la relación C:N más alta (35.7), por la incorporación de estiércol, aserrín y cáscara de semilla de calabaza como composta que dificulta la descomposición de la materia orgánica. Finalmente, los altos valores de la CIC de todos los sitios son considerados óptimos en cuanto a su fertilidad química (Cuadro 2) y la reacción química fue de moderada a fuertemente ácida de acuerdo con la NOM-RECNAT-2000 (Norma Oficial Mexicana, 2002).

En cuanto a elementos como el $\mathrm{K}^{+} \mathrm{y}$ los fosfatos se encontró que presentan concentraciones altas en algunos sistemas. En sistemas agroforestales, en donde se incorporan cenizas volcánicas, tanto en manejo orgánico (Sitio 5) como inorgánico (Sitio 6), los contenidos de $\mathrm{K}^{+}$se incrementaron hasta 109 y $98 \mathrm{cmol}$ (+) $\mathrm{kg}^{-1}$ respectivamente, en relación con el BMM que presentó $1.14 \mathrm{cmol}(+) \mathrm{kg}^{-1}$. El mismo comportamiento ocurrió con los fosfatos, donde los porcentajes se 
incrementaron de 2 a 3.5 veces más con el manejo orgánico (Sitio 3 y 7). En todos los sistemas analizados, el $\mathrm{Ca}^{2+}$ y $\mathrm{Mg}^{2}$ presentan valores bajos y muy bajos de acuerdo con la NOM-021-RECNAT-2000 (Norma Oficial Mexicana, 2002), por lo que es necesario su adición para mejorar la calidad de los suelos.

En la Figura 4 se ilustra de manera radial el comportamiento de algunas propiedades edáficas (Da, MOS, retención de humedad y CIC) entre los diferentes sistemas estudiados (forestal, agroforestal y agrícola). En términos generales, el sistema que más se diferencia del BMM, es el manejo agrícola; no obstante, si este último sistema incorpora un manejo agroforestal puede disminuir estas tendencias, como fue el caso del Sitio 4, el cual fue un sitio degradado.
También la incorporación de fertilizantes orgánicos e inorgánicos incrementa el contenido $\mathrm{N}_{\text {tot }}$, como ocurrió en $\operatorname{los}$ Sitio 2, 4 y 5.

\section{Índices de Calidad}

En cuanto a los índices de calidad de cada sistema se encontró que las propiedades que más se modificaron por el cambio de uso de suelo fueron: la morfología (estructura), el contenido de MOS y la humedad. Además, la adición de fertilizante ha incrementado el contenido de $\mathrm{N}_{\text {tot }}$, el cual determina la tasa de descomposición de la materia orgánica (Koranda et al., 2014) y la adición de cenizas incrementó el contenido de $\mathrm{K}^{+}$.
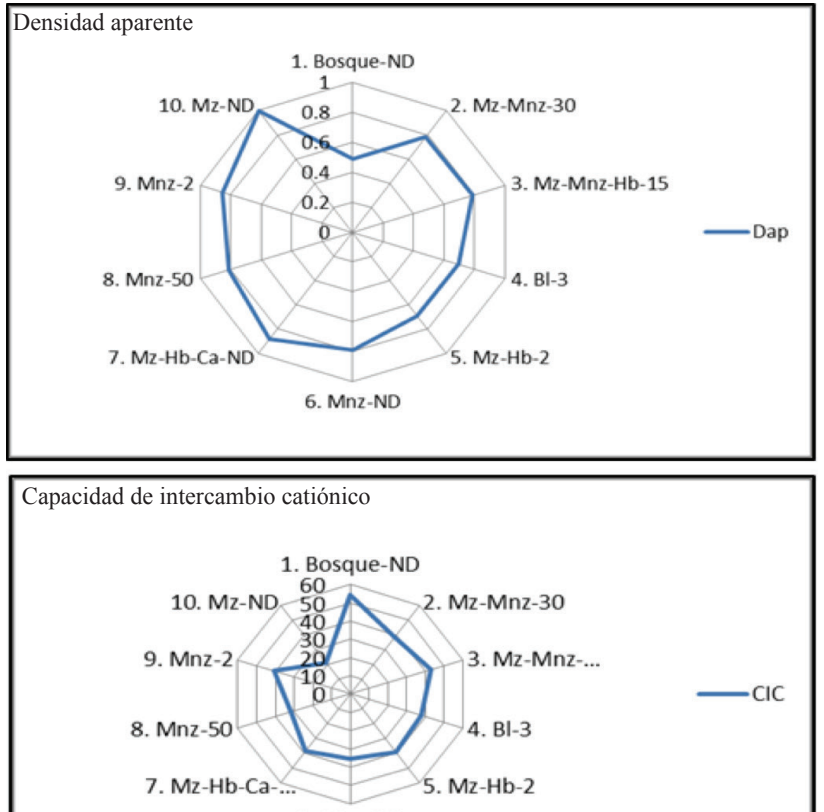

6. Mnz-ND

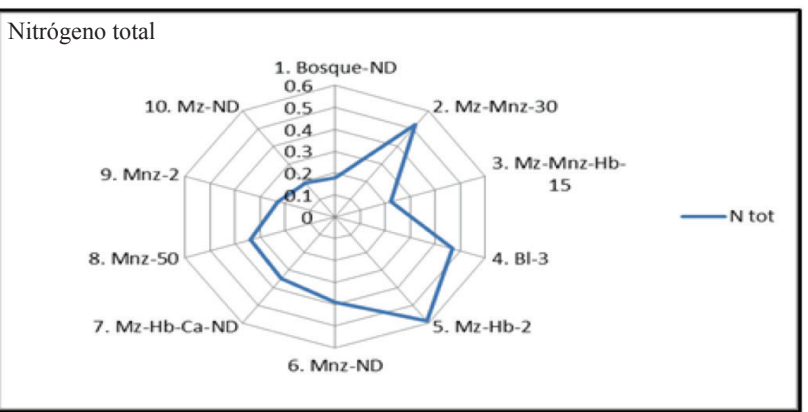

\% Retención de humedad

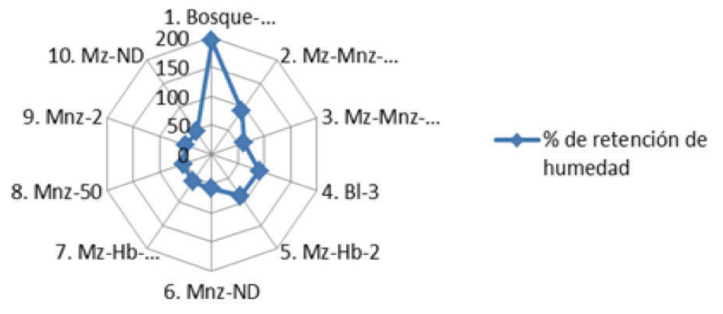

Materia orgánica

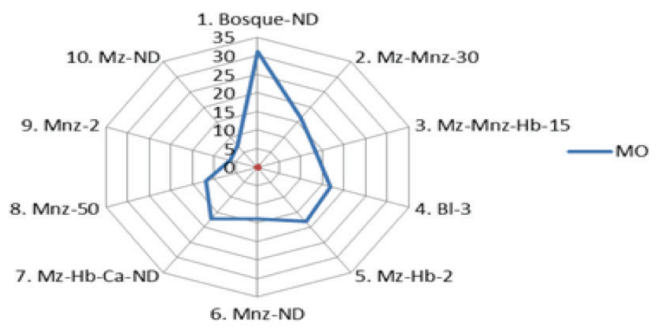

1. Bosque;

2. Mz-MNZ-30;

3. Mz-Mnz-Hb-15;

4. Bl-3;

5. Mz-Hb-2;

6. Mnz-ND;

7. Mz-Hb-Cal-ND;

8. Mnz-50;

9. Mnz-2;

10. Mz-ND.

$\mathrm{Mz}=$ maíz; $\mathrm{Mnz}=$ manzano $; \mathrm{Hb}=$ haba $; \mathrm{Cal}=$ calabaza $;$ $\mathrm{Bl}=$ arándanos. $\mathrm{ND}=$ no determinado. Los números después de las iniciales indican años de labranza.

Figura 4. Propiedades edáficas del bosque mesófilo de montaña (BMM) y los diferentes manejos agroforestales y agrícolas. 
Estos indicadores contrastan con los obtenidos por otros autores; por ejemplo, Cornwell (2014) encontró que del bosque nativo a la agroforestería (cacao, cacaoplátano, cacao-piña y cacao-pastizal) las propiedades que cambiaron fueron: $\mathrm{Da}, \mathrm{pH}, \mathrm{CIC}, \mathrm{Ca}$; mientras que las que no se modificaron fueron: $\mathrm{MOS}, \mathrm{N}, \mathrm{Mg}^{2+}, \mathrm{K}^{+}$ y P. En cuanto al tipo de labranza, Neupane y Thapa (2001) reportaron que en sistemas agroforestales contra sistemas de labranza convencional establecidos durante 4 años el $\mathrm{pH}, \mathrm{MOS}, \mathrm{N}_{\text {tot }}, \mathrm{P}_{\text {y K }}{ }^{+}$no reflejaron diferencias significativas.

Dada la variación de los indicadores de calidad en los sistemas agroforestales y agrícolas es necesario un entendimiento de los procesos edáficos, sobre todo para identificar procesos de degradación o recuperación de suelos (Orellana et al., 1997) y no sólo evaluar la capacidad de un suelo para llevar a cabo sus funciones (Larson y Pierce, 1994). Además, es conveniente seleccionar atributos o propiedades del suelo que sean más sensibles al manejo (Arshad y Martin, 2002), sus características y las condiciones ambientales donde se desarrollan (Cardoso et al., 2013).

\section{Consideraciones Finales}

Como información adicional, en los sistemas agroforestales se encontró que las huertas de manzano tienen problemas en el manejo de las plagas y deficiencias nutrimentales (Figuras $5 \mathrm{a}$ y b); además, las prácticas de incorporar aserrín y residuos de semillas al suelo (Figura 5c y d), pueden disminuir la disponibilidad del nitrógeno (Brady y Weil, 1999), y la calidad del suelo.

\section{CONCLUSIONES}

En el municipio de Zacatlán, Puebla, se presentaron tres tipos de sistemas en los Andosols: forestal, agroforestal yagrícola, con manejo orgánico e inorgánico y diferente tipo de labranza (cero y convencional). Los manejos orgánicos e inorgánicos con cualquier tipo de labranza han disminuido la calidad de los suelos en comparación con los suelos del bosque, tanto en sus propiedades físicas, químicas como morfológicas. El sistema agroforestal y agricultura con labranza mínima tienden a parecerse más al bosque mesófilo de montaña (BMM); además posiblemente están contribuyendo a la recuperación de suelos degradados. Caso contrario es el sistema agrícola con fertilización inorgánica, donde sus propiedades edáficas difieren hasta en $80 \%$ del BMM. Los índices de calidad de los tres sistemas estudiados que mejor representaron los cambios por uso del suelo fueron: morfología del suelo (estructura), el contenido de MOS y la retención de humedad, cuyos valores disminuyen de BMM a sistema agroforestal y agrícola. El $\mathrm{N}_{\text {tot }}, \mathrm{P}$ y $\mathrm{K}^{+}$están relacionados con la aplicación de fertilizantes y cenizas.

\section{LITERATURA CITADA}

Arriaga, L., J. M. Espinoza, C. Aguilar, E. Martínez, L. Gómez y E. Loa (coordinadores). 2000. Regiones terrestres prioritarias de México. Comisión Nacional para el Conocimiento y uso de la Biodiversidad. México.

Arshad, M. A. and S. Martin. 2002. Identifying critical limits for soil quality indicators in agro-ecosystems. Agric. Ecosyst. Environ. 88: 153-160.

Bautista-Cruz, A., J. Etchevers-Barra, R. F. del Castillo y M. C. Gutiérrez-Castorena. 2004. La calidad del suelo y sus indicadores. Ecosistemas 13: 90-97.
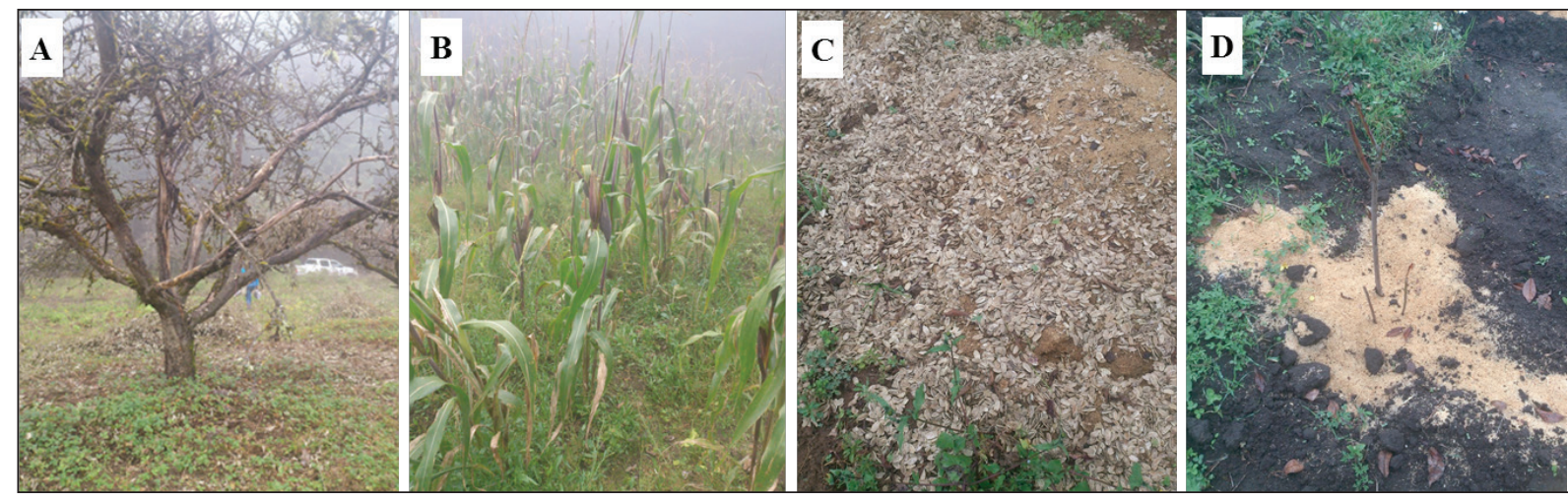

Figura 5. Problemas comunes en los sistemas agroforestales y agrícolas. A) Incidencia de plagas; B) Deficiencias nutrimentales en sistemas agrícolas. Malas prácticas agronómicas: C) aplicación de residuos de cáscara de semilla de calabaza; D) aplicación de aserrín. 
Brady, N. C. and R. R. Weil. 1999. The nature and properties of soil. Prentice Hall. Upper Saddle River, NJ, USA.

Bernal, M. P., J. A. Alburquerque, and R. Moral. 2009. Composting of animal manures and chemical criteria for compost maturity assessment. A review. Biores. Technol. 100: 5444-5453.

Bronick, C. J. and R. Lal. 2005. Soil structure and management: A review. Geoderma 124: 3-22.

Bullock, P., A. Jongerius, T. Tursina, N. Federoff, G. Stoops y U. Babel. 1985. Manual para la descripción micromorfológica de suelos. Trad. al español por M. C. Gutiérrez C. y C. A. Ortiz S. Programa de Edafología. Colegio de Postgraduados. Montecillo, Estado de México.

Cardoso, E. J. B. N., R. L. F. Vasconcellos, D. Bini, M. Y. H. Miyauchi, C. A. Santos, P. R. L. Alves, A. M. Paula, A. S. Nakatani, J. M. Pereira, and M. A. Nogueira. 2013. Soil health: Looking for suitable indicators. What should be considered to assess the effects of use and management on soil health? Sci. Agric. 70: 274-289.

Casanova-Lugo, F., L. Ramírez-Avilés, D. Parson, A. CaamalMaldonado, A. Piñeiro-Vázquez, and V. Díaz-Echeverría. 2016. Environmental services from tropical agroforestry systems. Rev. Chapingo Serie Cienc. For. Amb. 22: 269-284.

Cássaro, F. A. M., A. K. Borkowski, L. F. Pires, J. A. Rosa, and S. C. Saab. 2011. Characterization of a Brazilian clayey soil submitted to conventional and no-tillage management practices using pore size distribution analysis. Soil Tillage Res. 111: 175-179.

CATIE (Centro Agronómico Tropical de Investigación y Enseñanza). 2001. Módulos de enseñanza agroforestal. Funciones y aplicaciones de sistemas agroforestales. Turrialba, Costa Rica.

Cornwell, E. 2014. Effects of different agricultural systems on soil quality in Northern Limon province, Costa Rica. Rev. Biol. Trop. 62: 887-897.

Cuanalo de la C., H. 1990. Manual para la descripción de perfiles de suelo en el campo. Centro de Edafología, Colegio de Postgraduados. Montecillo, México.

Fa, J. E. and J. L. Morales.1993. Patterns of mammalian diversity in Mexico. pp. 319-365. In: T. P. Ramamoorthy, R. Bye, A. Lot, and J. Fa (eds.). Biological diversity of Mexico: Origins and distribution. Oxford University Press. Nueva York, NY, USA.

Gutiérrez-Castorena, Ma. del C., J. Reyes Ch. y B. Figueroa S. 1999. Desarrollo estructural en un Entisol bajo el sistema de labranza de conservación y tradicional. Terra Latinoamericana 17: 87-96.

Hammel, K. E. 1997. Fungal degradation of lignin. pp. 33-45. In: G. Cadish and K. E. Giller (eds.). Driven by nature: Plant litter quality and decomposition. $\mathrm{CAB}$ international. Oxon, UK.

IUSS Working Group WRB. 2015. Base referencial mundial del recurso suelo. 2014. Sistema internacional de clasificación de suelos para la nomenclatura de suelos y la creación de leyendas de mapas de suelos. Informes sobre recursos mundiales de suelos 106. FAO, Roma.

Koranda, M., C. Kaiser, L. Fuchslueger, B. Kitzler, A. Sessitsch, S. Zechmeister-Boltenstern, and A. Ritcher. 2014. Fungal and bacterial utilization of organic substrates depend on substrate complexity and $\mathrm{N}$ availability. FEMS Microbiol. Ecol. 87: 142-152.
Kooistra, M. J. and M. M. Pulleman. 2010. Features related to fauna activity. pp. 397-418. In: G. Stoops, V. Marcelino and F. Mees (eds.). Interpretation of micromorphological features of soils and regoliths. Elsevier. The Netherlands.

Krasilnikov, P., M. C. Gutiérrez-Castorena, R. J. Ahrens, C. O. Cruz-Gaistardo, S. Sedov, and E. Solleiro-Rebolledo. 2013. The soils of Mexico. Springer. Dordrecht, Netherlands.

Lal, R. 2002. Soil carbon dynamics in cropland and rangeland. Environ. Pollut. 116: 353-362.

Larson, W. E. and F. J. Pierce. 1994. The dynamics of soil quality as a measure of sustainable management. pp. 37-51. In: J. Doran, D. Coleman, D. Bezdicek, and A. Stewart. (eds.). Defining soil quality for a sustainable environment. SSSA Inc. Special Publication 35. Madison, WI, USA.

Martínez, M. L., O. Pérez-Maqueo, G. Vázquez, G. CastilloCampos, J. García-Franco, K. Mehltreter, M. Equihua and R. Landgrave. 2009. Effects of land use change on biodiversity and ecosystem services in tropical montane cloud forest of Mexico. For. Ecol. Manage. 258: 1856-1863.

McDaniel, P. A., D. J. Lowe, O. Arnalds, and Ch. L. Ping. 2012. Andisols. pp. 3-29, 33-48. In: P. M. Huang, Y. Li., M. E. Sumner (eds.). Handbook of soil sience: Properties and processes. CRC Press. Boca Raton, FL, USA.

Moreno-Calles, A. I., V. M. Toledo y A. Casas. 2013. Los sistemas agroforestales tradicionales de México: Una aproximación biocultural. Bot. Sci. 91: 375-398.

Muñoz-Villalobos, J. A., K. Oleschko-Lutkova, M. A. VelásquezValle, J. J. Velázquez-García, M. Martínez-Menes y B. Figueroa-Sandoval. 2011. Propiedades físicas de un Andosol mólico bajo labranza de conservación. Rev. Mex. Cienc. Agric. 1: 151-162.

Nair, P. K. R., B. M. Kumar, B. M., and V. D. Nair, 2009. Agroforestry as a strategy for carbon sequestration. J. Plant. Nutr. Soil Sci. 172: 10-23.

Neupane, R. P. and G. B. Thapa. 2001. Impact of agroforestry intervention on soil fertility and farm income under the subsistence farming system of the muddle hills, Nepal. Agric. Ecosyst. Environ. 84: 157-167.

Nogueira, M. A., U. B. Albino, O. Brandao-Junior, G. Braun, M. F. Cruz, B. A. Dias, R. T. D. Duarte, N. M. R. Gioppo, P. Menna, J. M. Orlandi, M. P. Raimam, L. G. L. Rampazo, M. A. Santos, M. E .Z. Silva, F. P. Vieira, J. M. D. Torezan, M. Hungria, and G. Andrade. 2006. Promising indicators for assessment of agroecosystems alteration among natural, reforested and agricultural land use in southern Brazil. Agric. Ecosyst. Environ. 115: 237-247.

Norma Oficial Mexicana NOM-021-RECNAT-2000. 2002. Norma Oficial Mexicana que establece las especificaciones de fertilidad, salinidad y clasificación de suelos. Estudios, muestreos y análisis. Diario Oficial de la Federación, 31 de diciembre de 2002. México, D. F.

Orellana, J. A., M. A. Pilati, and D. A. Grenón. 1997. Soil quality: An approach to physical state assessment. J. Sustain. Agric. 9: 91-108.

Orozco, H. E., V. Peña, R. Franco y N. Pineda. 2004. Atlas agrario ejidal del Estado de México. Cuadernos de Investigación, Núm. 34. UAEM. Toluca, Estado de México, México. 
Pagliai, M., N. Vignozzi, and S. Pellegrini. 2004. Soil structure and the effect of management practices. Soil Tillage Res. 79: 131-143.

H. Ayuntamiento de Zacatlán. 2011. Plan de Desarrollo Municipal 2011-2014. Zacatlán, Puebla, México.

Pires, L. F., J. A. R. Borges, J. A. Rosa, M. Cooper, R. J. Heck, S. Passoni, and W. L. Roque. 2017. Soil structure changes induced by tillage systems. Soil Till. Res. 165: 66-79.

Rahman, M. M., J. Tsukamoto, M. M. Rahman, A. Yoneyama, and K. M. Mostafa. 2013. Lignin and its effects on litter decomposition in forest ecosystems. Chem. Ecol. 29: 540-553.

Romero-Figueroa, J. C., J. Sánchez-Escudero, M. N. RodríguezMendoza y M. C. Gutiérrez-Castorena. 2015. Producción de vermicompost a base de rastrojo de maiz (Zea mays L.) y estiércol de bovino lechero. Agroproductividad 8: 52-59.

Sánchez, E. J., M. M. N. Rodríguez, R. C. V. Sánchez y L. F. Fernández. 2008. Abonos orgánicos. Ediciones Papiro Omega. México, D. F.

SEMARNAT-CP (Secretaría del Medio Ambiente y Recursos Naturales-Colegio de Postgraduados en Ciencias Agrícolas). 2002. Evaluación de la degradación de los suelos causada por el hombre en la República Mexicana a escala 1:250 000. Información complementaria a la Memoria Nacional. (CD Sitios de muestreo y datos analíticos). México, D. F.

Sharma, R., S. K. Chauhan, and A. M. Tripathi. 2016. Carbon sequestration potential in agroforestry system in India: An analysis for carbon project. Agroforest. Syst. 90: 631-644.

Shrestha, B. M., B. K. Sitaula, B. R. Singh, and R. M. Bajracharya. 2004. Soil organic carbon stocks in soil aggregates under different land use systems in Nepal. Nutr. Cycl. Agroecosys. 70: 201-213.

Spaccini, R., A. Zena, C. A. Igwe, J. S. C. Mbagwu, and A. Piccolo. 2001. Carbohydrates in water-stable aggregates and particle size fractions of forested and cultivated soils in two constrasting tropical ecosystems. Biogeochemistry 53: 1-22.

Stoops, G. 1983. Mineralogy and micromorphology of some andisols of Rwanda. pp. 150-165. In: F. H. Beinroth, H. Neel, and H. Eswaran (eds.). Proceedings of the Fourth International Soil Classification Workshop, Rwanda. Part 1, Papers. ABOSAGCD, Agricultural Editions 4. Rwanda

Stoops, G., E. Valvoulidou, and F. Monteiro. 2006. Micropedology, mineralogy and biology of soils derived from volcanic ash on Santorini (Greece). Geophys. Res. Abstr. 8: 10388.

Strong, N. and M. G. Jacobson. 2006. A case for consumer-driven extension programming: Agroforestry adoption potential in Pennsylvania. Agrofor. Syst. 68: 43-52.
Tapia-Vargas, M., M. Tiscareño-López, J. Stone, J. Oropeza-Mota, and M. Velázquez-Valle. 2001. Tillage system effects on runoff and sediment yield in hillslope agriculture. Field Crop Res. 69: 173-182.

Tavares Filho J. and J. D. Tessier. 2009. Characterization of soil structure and porosity under long-term conventional tillage and no-tillage systems. Rev. Bras. Ciênc. Solo 33: 1837-1844.

Torn, M. S., S. E. Trumbore, O. A. Chadwick, P. M. Vitousek, and D. M. Hendricks. 1997. Mineral control of soil organic carbon storage and turnover. Nature 389: 170-173.

Torralba, M., N. Fagerholm, P. J. Burgess, G. Moreno, and T. Plieninger. 2016. Do European agroforestry systems enhance biodiversity and ecosystem services? A meta-analysis. Agric. Ecosyst. Environ. 230: 150-161.

USDA (United States Department of Agriculture). 2014. An overview of agroforestry, Agroforestry Note \#1, General \#1. Washington, DC, USA.

Van Reeuwijk, L. P. 2003. Procedimiento para análisis de suelos. Trad. al español por M. C. Gutiérrez Castorena, C. A. Tavares E. y C. A. Ortiz Solorio. Colegio de Postgraduados. Montecillo, Estado de México.

Velásquez-Valle M. A., M. R. Martínez-Menes, M. BravoEspinosa, J. J. Velázquez-García, K. N. Potter y M. TiscareñoLópez. 2006. Erosionabilidad acanalada de un Andosol en dos sistemas de labranza en la Meseta Tarasca, Michoacán. Terra Latinoamericana 24: 119-123.

Velázquez-García, J. J., K. Oleschko, J. A. Muñoz-Villalobos, M. A. Velásquez-Valle, Y. Girón-Ríos, M. Martínez-Menes, y B. Figueroa-Sandoval. 2007. El color del Andosol como un indicador de su calidad física bajo el manejo. Terra Latinoamericana 25: 1-8.

Villaseñor, J. L. 2010. El bosque húmedo de montaña en México y sus plantas vasculares. Catálogo florístico-taxonómico. Comisión Nacional para el Conocimiento y Uso de la Biodiversidad-UNAM. México, D. F.

Wilson, M. H. and S. T. Lovell. 2016. Agroforestry-The next step in sustainable and resilient agriculture. Review. Sustainability 8: 574. doi:10.3390/su806057.

Young, A. 1989. Agroforestry for soil conservation (Science and practice of agroforestry). CAB International. Oxford University Press. Wallingford, UK.

Young, A. 1997. Agroforestry for soil management. CAB International. Oxford University Press. Wallingford, UK. 